\title{
Nurse Versus Clinician Symptomatic Adverse Events Assessment Based on Patient-Reported Outcomes Version of the Common Terminology Criteria for Adverse Events (PRO-CTCAE): Results of a Questionnaire-Based Study
}

\section{Lei Liu}

Hebei Medical University Fourth Affiliated Hospital and Hebei Provincial Tumor Hospital Jian-xin Wang

Hebei Medical University Fourth Affiliated Hospital and Hebei Provincial Tumor Hospital

\section{Li-na Zhang}

Hebei Cancer Institute: Hebei Medical University Fourth Affiliated Hospital and Hebei Provincial Tumor Hospital

\section{Yan-shou zhang}

Hebei Cancer Institute: Hebei Medical University Fourth Affiliated Hospital and Hebei Provincial Tumor Hospital

\section{Feng-xia Liu}

Hebei Cancer Institute: Hebei Medical University Fourth Affiliated Hospital and Hebei Provincial Tumor Hospital

\section{Lin Kang}

Hebei Medical University Fourth Affiliated Hospital and Hebei Provincial Tumor Hospital

\section{Xin-le Wang}

Hebei Medical University Fourth Affiliated Hospital and Hebei Provincial Tumor Hospital

Zhanlun Liu ( $\nabla 34490149 @ q q . c o m$ )

Hebei Provinvial Hosptial of Traditional Chinese Medcine https://orcid.org/0000-0002-8105-9068

\section{Research article}

Keywords: Nurses' role, PRO-CTCAE, Consistency, Questionnaire, Subjective adverse events

Posted Date: April 5th, 2021

DOI: https://doi.org/10.21203/rs.3.rs-390542/v1

License: (1) (1) This work is licensed under a Creative Commons Attribution 4.0 International License. Read Full License 


\section{Abstract}

Background: Monitoring adverse Events (AEs) is a standard practice for clinician to assess the overall effect of anti-cancer treatment on patients. However, studies have found clinicians have difficulty in capturing nuance aspects of patient-centered AEs. We aimed to explore the differences between nurses and clinicians in term of subjective AEs assessment and discuss the nurses' role in subjective AEs assessment.

Methods: From April and July, 2020, a questionnaires based study was conducted in Day Chemotherapy ward of Breast Center in Hebei Cancer Hospital. Six common subjective AEs were assessed by nurses and clinicians utilizing Common Terminology Criteria for Adverse Events (CTCAE). Patients needed to self-report their own subject AEs by filling up a Simplified Chinese version of PRO-CTCAE containing the same AEs. Results from the three different pairs (Nurse/Patient, Nurse/ Clinician, and Clinician/Patient) were collected and analyzed. Chi-square test and kappa coefficient were utilized to analysis the differences and consistency among different pairs.

Results: of 417 patients were asked to participate in the study, 384 pairs surveys were collected. Nurses detected more AEs than clinicians, and the differences were statistically significant ( $P \otimes 0.00)$. Nurse/patient pair scored the highest agreement in terms of reporting rate and toxicity grade for most of AEs except the toxicity grade for the frequency of vomiting and pain which were as consistent as nurse/clinician.

Conclusions: The results of this study support nurses can be successfully enrolled in the assessment of subjective AEs because of the greater ability to elicit more patient-centered information than clinicians.

\section{Background}

Cancer incidence and mortality are growing rapidly, and there would be an estimated 18.1 million new cancer cases in 2018 according to the report launched by the International Agency for Research on Cancer [1]. Chemotherapy is considered an effective method to stop tumor progression. However, it also has the risk of substantial adverse events (AEs). Although AEs are inevitable especially when patients go through chemotherapy, accurate and timely reporting allow for risks to be minimized. AEs assessment is therefore not only the focus of clinical trials, but also important to be routinely assessed in the clinical setting, since AEs have vital influence on patients' quality of life.

AEs assessment has become structured in recent years. The sensitive tools for AEs assessment is CTCAE developed by the National Cancer Institute $(\mathrm{NCl})$. CTCAE classifies all AEs into three categories: AEs based on laboratory results, measurable AEs and patient-centered AEs. Previous studies have found that clinicians under-detected and under-grade subjective AEs [2,3]. Inaccurate reporting of toxicity may serve as a wrong single for clinicians to make inappropriate treatment decisions, which may delay patients' treatment and influence patients' quality of life. Therefore, $\mathrm{NCl}$ has worked to initiate a patient-self reported tool which is called PRO-CTCAE to assess patient-centered AEs and improve the accuracy of clinicians' reporting [4]. Patients' role in AEs assessment have been affirmed by many studies [5-11]. Our previous study has proved that patients play a vital role in detecting subjective AEs [12]. However, how to better combine those independent rating sources together and make full use of the information still lack of strong research 
evidence. Assessing AEs by assigning them to expertise in the corresponding filed according to the nature of the AEs might be a way to improve the accuracy of reporting. Therefore, we hypothesized that nursing staff could be an alternative way to assess subjective AEs due to their educational background and better communication skill. This study is therefore to compare the differences of subjective adverse event assessment between nurses and clinicians based on PRO-CTCAE and to discuss whether nurses have the ability to assess patients' subjective AEs.

\section{Methods}

\section{Material and Method}

From April 2020 to July 2020, a single center questionnaire-based study was conducted in Day Chemotherapy ward of the Breast Center in the Fourth Hospital of Hebei Medical University. Breast cancer patients who are undergone chemotherapy were enrolled in our study, while patients who are illiterate, having hearing and visual impairment were restricted to this study. All enrolled patients were invited to selfreport their AEs by completing the PRO-CTCAE questionnaire before this round treatment start, and they were asked to complete the questionnaire only once. No specific assistance were provided by medical staff when patients completed their questionnaires, while they were always available to explain the questions of clarification. General information of enrolled patients, the reporting rate and toxicity grade of all subjective AEs for the past 7 days were obtained from all patients.

Before assessing patients' subjective AEs, nurses and clinicians with GCP (Good Clinical Practice, GCP) were asked to attend a two weeks training as regards $A E$ assessment. The study only enrolled nurses and clinicians who completed the course and passed the test for AEs assessment. The same subjective AEs specified in the patient questionnaire were assessed by nurses and clinicians using CTCAE scale version 5.0. Every enrolled patient was requested to describe every subjective AEs again when they were assessed by nurses and clinicians.

We realized that it is impossible to assess all subjective AEs listed in PRO-CTCAE at one time, as it may increase patients' burden to complete the questionnaire, which, in turn, affecting the quality of the study. On the other hand, the results of the study will also be affected by assessing AEs with low incidence rate. Therefore, a pilot study was conducted first in targeted patients to select AEs with high reporting rate [12]. Nausea, vomiting, diarrhea, pain, constipation and fatigue were finally selected to be the targeted symptoms. Nurses, clinicians and patients need to assess all above AEs according to the description of CTCAE version 5.0 and simplified Chinese PRO-CTCAE version 1 accordingly. The results of the pilot study, being used to extract AEs with high incidence and estimating the sample size, are not included in the final study analysis.

In order to control the bias of the study, we requested every nurse-clinician-patient must complete the questionnaires on the same day, and the integrity of all questionnaires were checked at time of completion in case of any items missing. All three parties were not allowed to access each other's answers. This study 
was conducted with the approval from the Ethics committee of the Hebei University of Chinese Medicine, and written informed consent was provided from all participants.

\section{CTCAE and PRO-CTCAE questionnaire}

CTCAE is the standard approach for medical staff to assess and document AEs after cancer treatment. The current version of CTCAE (version 5) includes 790 items derived from objective AEs and subjective AEs. Every item was assessed by unique description, with a scale of 1-5 to express the severity of the AEs. CTCAE is more clinician centered and emphasized more on laboratory AEs. PRO-CTCAE was created as a companion of CTCAE by $\mathrm{NCl}$ through extracting all subjective AEs from CTCAE to improve the accuracy and reliability of clinicians' AEs assessment. PRO-CTCAE comprises 124 items representing 78 subjective symptoms. Every subjective AEs were elicited using between one to three different aspects: the severity of subjective AEs, the frequency of subjective AEs, and the interference of subjective AEs[4]. PRO-CTCAE is more patient centered and focused more on impacts on patients' emotion and function.

The Chinese versions of the two scales were developed through cross-cultural adaption. Methods used to translate and linguistically validate tools follow the Principles of Good Translation and Cultural Adaptation Practice as articulated by ISPOR. The simplified Chinese versions were tested in China and Singapore.

\section{Statistical method}

Statistical analysis was performed using SPSS 25.0 software. The count data were expressed as ( \pm s) or medians and quartiles. The measurement data were expressed as frequency and percentage. Chi-square test and Cohen's kappa coefficient were utilized to compare the differences and the consistency in term of reporting rate and toxicity grade among three pairs. As CTCAE and PRO-CTCAE questionnaire has different numbers of items and response option, therefore, we have matched all response option to be identical pairs when toxicity grade was compared[13]. Minimum sample size $(n=384)$ was calculated based on the results of pilot study.

\section{Results}

Between April and July, 2020, 417 consecutive breast cancer patients were invited to participate in the study. Of these, 7 patients were illiterate and 5 patients refused to participate, resulting in total of 405 patients enrolled in our study. During the process of data sorting, 21 pairs questionnaires which not completed on the same day were eliminated. The remaining 384 pairs questionnaires were available for analysis. Table 1 shows baseline characteristics of patients in completed pairs. 3 clinicians (master degree) and 4 nurses (2 with master degree and 2 with bachelor degree) who have obtained the national GCP certificate and the professional qualification certificate of relevant specialties were enrolled in our study. 
Table 1

Main demographic and clinical characteristics of patients

\begin{tabular}{|ll|}
\hline & $\mathbf{n}(\%)$ \\
\hline Age & \\
\hline median, range & $50.5(24-75)$ \\
\hline Male & $2(0.5 \%)$ \\
\hline Female & $382(99.5 \%)$ \\
\hline Stage of Disease & \\
\hline Late Stage & \\
\hline Early Stage & \\
\hline Education Level & $26(6.8 \%)$ \\
\hline High school and Above & $358(93.2 \%)$ \\
\hline Primary school to High school & $227(59.1 \%)$ \\
\hline Time & \\
\hline median, range & $414.7 S(48 S-5805 S)$ \\
\hline
\end{tabular}

* Late stage:III, IV, Metastatic; ${ }^{* *}$ Early stage: 0, I, II;

\section{Incidence of subjective AEs}

According to whether there was an AE present, all collected data were categorized into two group: no AEs present group (For AEs with grade 0 ) and a AEs present group (For AEs with grade 1-5). The reporting rates for all AEs were analyzed in accordance with frequency, severity and life interference. Table 2 indicates the overall reporting rate for nurses, clinicians and patients. Patients tended to report the most AEs. Nurses reported more AEs than clinicians, and the differences were statistically significant ( $P ख 0.00$, Table 3). Cohen's kappa coefficient was utilized to analysis the consistency among different pairs, and the results shows the reporting rate for all observed AEs in nurse/patient pair was consistently higher than the other two pairs (Fig. 1). 
Table 2

Reporting rate of subjective AEs completed by nurse, clinicians and patients

\begin{tabular}{|c|c|c|c|c|c|c|c|c|c|}
\hline \multirow[t]{2}{*}{ Adverse events } & \multicolumn{3}{|c|}{ Nurses } & \multicolumn{3}{|c|}{ Clinicians } & \multicolumn{3}{|c|}{ Patients } \\
\hline & $\begin{array}{l}\text { No } \\
\text { AE }\end{array}$ & $\begin{array}{l}\text { An } \\
A E\end{array}$ & $\begin{array}{l}\text { Reporting } \\
\text { rate }\end{array}$ & $\begin{array}{l}\text { No } \\
\text { AE }\end{array}$ & $\begin{array}{l}\mathrm{An} \\
\mathrm{AE}\end{array}$ & $\begin{array}{l}\text { Reporting } \\
\text { rate }\end{array}$ & $\begin{array}{l}\text { No } \\
\mathrm{AE}\end{array}$ & $\begin{array}{l}\text { An } \\
A E\end{array}$ & $\begin{array}{l}\text { Reporting } \\
\text { rate }\end{array}$ \\
\hline Nausea/Frequency & 104 & 280 & 72.9 & 188 & 196 & 51.0 & 58 & 326 & 84.9 \\
\hline Nausea/ Severity & 104 & 280 & 72.9 & 188 & 196 & 51.0 & 73 & 311 & 81.0 \\
\hline Vomiting/ Frequency & 180 & 204 & 53.1 & 235 & 149 & 38.8 & 128 & 256 & 66.7 \\
\hline Vomiting/Severity & 180 & 204 & 53.1 & 235 & 149 & 38.8 & 144 & 240 & 62.5 \\
\hline Diarrhea/Frequency & 196 & 188 & 49.0 & 327 & 57 & 14.8 & 160 & 224 & 58.3 \\
\hline Fatigue/Severity & 101 & 283 & 73.7 & 292 & 92 & 24.0 & 30 & 353 & 92.2 \\
\hline $\begin{array}{l}\text { Fatigue/Life } \\
\text { Interference }\end{array}$ & 101 & 283 & 73.7 & 292 & 92 & 24.0 & 53 & 331 & 86.2 \\
\hline Pain/Frequency & 155 & 229 & 59.6 & 243 & 141 & 36.7 & 82 & 302 & 78.6 \\
\hline Pain/Severity & 155 & 229 & 59.6 & 243 & 141 & 36.7 & 103 & 281 & 73.2 \\
\hline $\begin{array}{l}\text { Pain/Life } \\
\text { Interference }\end{array}$ & 155 & 229 & 59.6 & 243 & 141 & 36.7 & 133 & 251 & 65.4 \\
\hline Constipation/severity & 256 & 128 & 33.3 & 358 & 26 & 6.8 & 184 & 184 & 47.9 \\
\hline
\end{tabular}

Table 3

Comparison of reporting rate between Nurse and Clinician

\begin{tabular}{|c|c|c|c|c|c|c|}
\hline \multirow[t]{2}{*}{ Adverse Events } & \multicolumn{2}{|l|}{ Nurse } & \multicolumn{2}{|c|}{ Clinician } & \multirow[t]{2}{*}{$x^{2}$} & \multirow[t]{2}{*}{$P$} \\
\hline & No AE & An $\mathrm{AE}$ & No $A E$ & An $\mathrm{AE}$ & & \\
\hline Nausea & 104 & 280 & 188 & 196 & 38.988 & 0.000 \\
\hline Vomiting & 180 & 204 & 235 & 149 & 89.280 & 0.000 \\
\hline Diarrhea & 196 & 188 & 327 & 57 & 102.858 & 0.000 \\
\hline Fatigue & 101 & 283 & 292 & 92 & 190.110 & 0.000 \\
\hline Pain & 155 & 229 & 243 & 141 & 40.387 & 0.000 \\
\hline Constipation & 256 & 128 & 358 & 26 & 80.503 & 0.000 \\
\hline
\end{tabular}

\section{Toxicity grade of subjective AEs}

Table 4 indicates the type of toxicity according to grade reported by nurses, clinicians and patients. The highest score for nurses and clinicians for six subjective AEs was 2 , while the highest score for patients was 4. Weighted kappa coefficient was calculated as a measurement to analyze the agreement among different pairs. The consistency for nurse/patient pair scored the highest for almost AEs among the three pairs 
except for the frequency of vomiting and pain which were as consistent as nurse/clinician pair (Fig. 2). We further compared the toxicity reported by nurses and clinicians through chi-square test, and we found there were no statistically significant in the severity of nausea $\left(\chi^{2}=1.062, P=0.303\right)$, vomiting $\left(\chi^{2}=2.656, P=\right.$ 0.103), diarrhea $\left(\chi^{2}=2.342, P=0.126\right)$, pain $\left(\chi^{2}=2.494, P=0.114\right)$ and constipation $\left(\chi^{2}=0.347, P=0.556\right)$, and there was statistically significant in the severity of fatigue $\left(\chi^{2}=5.083, P=0.024\right)$ (See Table 5 ).

Table 4

Grades according to questionnaires completed by nurses, clinicians and patients

\begin{tabular}{|llllllllllll|}
\hline Adverse Event & \multicolumn{1}{l}{ Nurse } & \multicolumn{1}{c}{ Clinician } & \multicolumn{7}{l|}{ Patient } \\
& $\mathbf{0}$ & $\mathbf{1}$ & $\mathbf{2}$ & $\mathbf{0}$ & $\mathbf{1}$ & $\mathbf{2}$ & $\mathbf{0}$ & $\mathbf{1}$ & $\mathbf{2}$ & $\mathbf{3}$ & $\mathbf{4}$ \\
\hline Nausea/Frequency & 104 & 198 & 82 & 188 & 147 & 49 & 58 & 140 & 134 & 44 & $\mathbf{8}$ \\
\hline Nausea/Severity & 104 & 198 & 82 & 188 & 147 & 49 & 73 & 185 & 97 & 19 & 10 \\
\hline Vomiting/Frequency & 180 & 146 & 58 & 235 & 118 & 31 & 128 & 128 & 105 & 21 & 2 \\
\hline Vomiting/Severity & 180 & 146 & 58 & 235 & 118 & 31 & 144 & 142 & 74 & 18 & 6 \\
\hline Diarrhea/Severity & 196 & 125 & 63 & 327 & 44 & 13 & 160 & 119 & 83 & 19 & 3 \\
\hline Fatigue/Severity & 101 & 186 & 97 & 292 & 72 & 20 & 30 & 180 & 147 & 24 & 3 \\
\hline Fatigue/Life interference & 101 & 186 & 97 & 292 & 72 & 20 & 53 & 167 & 137 & 21 & 6 \\
\hline Pain/Frequency & 155 & 158 & 71 & 243 & 108 & 33 & 82 & 119 & 130 & 45 & 8 \\
\hline Pain/Severity & 155 & 158 & 71 & 243 & 108 & 33 & 103 & 166 & 89 & 22 & 4 \\
\hline Pain/Life interference & 155 & 158 & 71 & 243 & 108 & 33 & 133 & 133 & 2 & 24 & 2 \\
\hline Constipation/Severity & 256 & 95 & 32 & 358 & 8 & 8 & 200 & 131 & 38 & 12 & 3 \\
\hline
\end{tabular}

Table 5

Comparison of toxicity reported by Nurse and Clinician

\begin{tabular}{|c|c|c|c|c|c|c|c|c|c|c|c|c|}
\hline \multirow[t]{2}{*}{ Group } & \multicolumn{2}{|c|}{ Nausea } & \multicolumn{2}{|c|}{ Vomiting } & \multicolumn{2}{|c|}{ Diarrhea } & \multicolumn{2}{|c|}{ Fatigue } & \multicolumn{2}{|l|}{ Pain } & \multicolumn{2}{|c|}{ Constipation } \\
\hline & 1 & 2 & 1 & 2 & 1 & 2 & 1 & 2 & 1 & 2 & 1 & 2 \\
\hline Nurse & 147 & 49 & 118 & 31 & 44 & 13 & 72 & 20 & 108 & 33 & 18 & 8 \\
\hline Clinician & 198 & 82 & 146 & 58 & 125 & 63 & 186 & 97 & 158 & 71 & 95 & 32 \\
\hline$\chi^{2}$ & 1.062 & & 2.656 & & 2.342 & & 5.08 & & 2.494 & & 0.347 & \\
\hline$P$ & 0.303 & & 0.103 & & 0.126 & & 0.02 & & 0.114 & & 0.556 & \\
\hline
\end{tabular}

\section{Discussion}


Our study explores the differences between nurses and clinicians on subjective AEs assessment based on PRO-CTCAE, and discusses whether nurses have the ability to assess patients' subjective AEs. The results demonstrate that nurses tend to report more AEs than clinicians $(P \otimes 0.00)$ and the consistency between nurses and patients was higher than that of the other two pairs. Coefficient of agreement for reporting rate between nurses and patients was quite good for most of AEs ( $k$ ranged from 0.546 to 0.813 ), except for fatigue which was poorly consistent for all pairs. Kappa value decreased for all pairs when toxicity grade was considered. Although the agreement between nurse/patient pair was the highest for most of the items, the kappa value [ranged from $0.294(0.228-0.360)$ to $0.701(0.655-0.747)$ ] indicated fair to substantial agreement. The lowest agreement was recorded in clinician/patient pair [ $\mathrm{k}$ ranged from $0.067(0.042-0.091)$ to $0.399(0.342-0.455)$ ]. Besides, it is intermediate in nurse/clinician pair [ $\mathrm{k}$ ranged from $0.145(0.096-$ $0.194)$ to $0.497(0.426-0.568)]$. Therefore, the results of this study indicate that nurses are more accurate in subjective AEs assessment than clinicians in terms of the consistency of AEs reporting rate and toxicity grade.

The fact that clinicians tended to report fewer AEs have been proved by many studies.[14-16] However, previous studies used to collect patients' data through extracting information from quality of life (QOL) questionnaires. This data extraction and conversion may directly lead to misunderstand and lose of information. Therefore, PRO-CTCAE was adopted in our study to avoid reducing information omission and facilitate an intuitive and sensitive self-assessment. The possible reasons for higher consistency between nurses and patients can be explained by different educational background. The American Nurses Association (ANA) emphasizes that nurses have mastered the assessment skills through professional knowledge. Previous studies [17-18] have shown that nurses have the ability to collect patients' data from patient's perspective and advocate for patients' rights. Nurses viewed themselves as patient advocates and patients' safety take precedence over all other interests [19]. On the contrary, clinicians put much focus on therapeutic effect, and by measuring treatment-related AEs to identify more effective treatment approach. Therefore, symptoms generally considered side-effect of disease or emotional distress often be neglected. For example, AEs like fatigue, which most likely caused by emotional distress, can be easily ignored by clinicians. In our study, more than $90 \%$ of patients complained of fatigue, and six patients considered fatigue had seriously affected their daily life. $73.7 \%$ nurses reported this $A E$, while only $24.0 \%$ clinicians reported it. It is true that only measuring AEs caused by treatments is not enough. Subjective AEs are very important and cannot be ignored, as they are mostly the emotional expression of patients' cognition for themselves and disease. We can measure patients' level of satisfaction with the therapy through evaluating any improvements on patients' quality of life and patients' perception of health[20-23].

An alternative explanation for the higher consistency between nurses and patients could be nurses' greater communication skill. In clinical setting, some patients are reluctant to report AEs, due to the fact that the more and the severe AEs they have, the higher chance of drug dosage adjusting [8], which in turn, may eventually affect therapeutic effect [24]. In our study, the under-reporting rate for clinicians ranged from 49\%-93.2\%, While clinicians assessed patients' AEs based on patients' description. Therefore, we believed that patients' wording may have some impact on clinicians' judgement. Besides, clinicians usually assess patients' AEs during medical visit, usually only about 10 minutes; this limited window is not sufficient for patients to fully convey their issues. Nurses tend to have a greater communication skill to elicit more 
information when patients tend to omit. What's more, medication care is part of nurse's daily work. Nurses need to closely observe and report all reactions of patients even if more likely due to disease rather than due to anti-tumor treatment. Therefore, nurses can be considered as the first healthcare professionals to work with patients when they experience any discomfort. Nurses are often the first healthcare professional to work with research patients on a new intervention, drug or device [25].

Kappa value decreased for all pairs when grade toxicity was considered. And we noticed that the highest score for nurses and clinicians was 2 , while the highest score for patients was 4 . As noted, medical decisions are made when serious AEs with grade 3 or higher are reported. In our study, there were no changes on medical decisions due to severe AEs. A possible explanation for grade difference in our study can be explained by different assessing tools we utilized. CTCAE specifies the frequency of AEs occurred, and each corresponding treatment measures were taken according to scoring level. Therefore, only when AEs meet the CTCAE criterion can be grade as 3 or higher. While PRO-CTCAE focus on patients' subjective perception on health and quality of life. However, we believed that utilizing different assessing tools is inevitable due to different educational background between medical team and patient. And in our study, we tried to minimize this impact through utilizing most sensitive tools for medical team and patients, and PROCTCAE was developed form the same content of CTCAE. On this basis, we further compared the grade differences between nurses and clinicians; we found there were no statically differences between nurses and clinicians for most of AEs except for fatigue which may attribute to different reporting rate for nurses and clinicians (73.7\% vs $24.0 \%$ ). Therefore, we believed nursing staff have the ability to grade patients' subjective AEs independently after gone through specific training as regards of AEs assessment.

We do have some limitations. The result of this study represents a relatively small sample size from single center consisting a single disease type patient with a relatively good performance status. The results of this study should be conformed in a large and multi-center study.

\section{Conclusions}

Our analysis suggests nursing staff have greater ability to assess patient's subjective AEs compared to clinician. AEs assessment is vital for patients' medical treatment and quality of life, enrolling of nursing staff into AEs assessment could be an alternative way to improve the accuracy of AEs assessment and help clinicians to establish the anti-tumor treatments' real advantages according to patients' perception of quality of life and prolong patients' life span eventually.

\section{Abbreviations}

AEs: Adverse events; PRO-CTCAE:Patient-Reported Outcomes version of the Common Terminology Criteria for Adverse Events; CTCAE: Common Terminology Criteria for Adverse Events;PRO: Patient-Repored Outcomes; GCP: Good Clinical Practice.

\section{Declarations}




\section{Ethics approval and consent to participate}

All patients provided written informed consent before participating. The study was approved by the Ethics Committee of University of Traditional Chinese Medicine (YXLL20191227).

\section{Consent for publication}

Not applicable.

\section{Availability of data and materials}

All data used and analysed in our current study available from the corresponding author on reasonable request.

\section{Competing interests}

The authors declare that they have no conflict of interest.

\section{Funding}

No funding was obtained for this study.

\section{Authors' contribution}

ZLL: project administration, conceptualization, methodology, investigation, formal analysis, data management, writing the original draft. XW: data verifying, nursing team management, figures verifying. LL: conceptualization, formal analysis, validation, writing, reviewing, and editing. XL, LK: data management, validation, investigation. NZ, SZ: conceptualization, supervision, writing, reviewing, and editing. LW: methodology, software, resources.

All authors have read and approved the manuscript.

\section{Acknowledgement}

The authors thank the participation of all enrolled patients, and thank you for the contributions of all clinicians and nurses.

\section{Authors' Information}

Hebei Provincial Hospital of Traditional Chinese Medicine, Shijiazhuang, Hebei Province, 050011, China Zhanlun Liu

\section{Breast Center, the $4^{\text {th }}$ Hospital of Hebei Medical University, Shijiazhuang, Hebei Province, China}

Lei Liu, Lina Zhang, Yanshou Zhang, Fengxia Liu, Lin Kang, Xinle Wang, Jianxin Wang 


\section{References}

1. Guy RC. International Conference on Harmonization. Encyclopedia of Toxicology. 2014;1070-1072. https://doi.org/10.1016/B978-0-12-386454-3.00861-7.

2. Ebile AW, Ateudjieu J, Yakum MN. Assessing the detection, reporting and investigation of adverse events in clinical trial protocols implemented in Cameroon: a documentary review of clinical trial protocols. BMC Medical Ethics. 2015;16(1):67. https://doi.org/10.1186/s12910-015-0061-5.

3. Banerjee AK., Okun S, Edwards IR., et al. Patient-Reported Outcome Measures in Safety Event Reporting: PROSPER Consortium guidance. Drug Safe. 2013;36(12):1129-1149. https://doi.org/10.1007/s40264013-0113-z.

4. Basch E, Reeve BB, Mitchell SA, et al. Development of the National Cancer Institute's patient-reported outcomes version of the common terminology criteria for adverse events (PRO-CTCAE). Journal of the National Cancer Institute. 2014;106(9),dju244. https://doi.org/10.1007/s11136-013-0470-1.

5. Basch E, Abernethy AP, Mullins CD, et al. Recommendations for Incorporating Patient-Reported Outcomes into Clinical Comparative Effectiveness Research in Adult Oncology. Journal of Clinical Oncology. 2012;30(34):4249-4255. https://ascopubs.org/doi/10.1200/JC0.2012.42.5967.

6. Storey DJ, Waters RA, Hibberd CJ, et al. Clinically relevant fatigue in cancer outpatients: the Edinburgh Cancer Centre symptom study. Annals of Oncology. 2007;18(11):1861-1189. https://doi.org/10.1093/annonc/mdm349.

7. Wilson A, Dowling $A$, Abdolell $M$, et al. Perception of quality of life by patients, partners and treating physicians. Quality of Life Research. 2009;9(9):1041-1052. https://doi.org/10.1023/A:1016647407161.

8. Pakhomov S, Jacobsen SJ, Chute CG, et al. Agreement between patient-reported symptoms and their documentation in the medical record. American Journal of Managed Care. 2008;14(8):530-539.PMCID: PMC2581509.

9. Atkinson TM., Ryan SJ, Bennett AV, et al. The association between clinician-based common terminology criteria for adverse events (CTCAE) and patient-reported outcomes (PRO): a systematic review. Supportive Care in Cancer. 2016;24(8):3669-3676. https://doi.org/10.1007/s00520-016-3297-9.

10. Chung AE, Shoenbill K, Mitchell SA, et al. Patient free text reporting of symptomatic adverse events in cancer clinical research using the National Cancer Institute's Patient-Reported Outcomes version of the Common Terminology Criteria for Adverse Events (PRO-CTCAE). Journal of the American Medical Informatics Association. 2019;26(4),276-285. https://doi.org/10.1093/jamia/ocy169.

11. Kawaguchi T, Azuma K, Sano M, et al. The Japanese version of the National Cancer Institute's patientreported outcomes version of the common terminology criteria for adverse events (PRO-CTCAE): psychometric validation and discordance between clinician and patient assessments of adverse events. Journal of Patient-Reported Outcome. 2017;2(1):1-13. https://doi.org/10.1186/s41687-0170022-5.

12. Liu L, Suo T, Shen Y, et al. (2020). Clinicians versus patient's subjective adverse events assessment: based on patient-reported outcomes version of the common terminology criteria for adverse events 
(PRO-CTCAE). Quality of Life Research. 2020;29(10):3009-3015. https://doi.org/10.1007/s11136-02002558-7.

13. Quinten C, Maringwa J, Gotay CC, et al. Patient Self-Reports of Symptoms and Clinician Ratings as Predictors of Overall Cancer Survival. JNCI Journal of the National Cancer Institute. 2011;103(24):18511858. https://doi.org/10.1093/jnci/djr485,

14. Ingham J, Portenoy RK. The measurement of pain and other symptoms. In: Doyle D, Hanks G, MacDonald N, eds. Oxford Textbook of Palliative Medicine. Oxford: Oxford University Press; 2011. p.203-219.

15. Patrick DL, Ferketich SL, Frame PS, et al. National Institutes of Health State-of-the-Science Conference Statement: symptom management in cancer: pain, depression, and fatigue. J Natl Cancer Inst. 2003;95(15):1110-1117. https://doi.org/10.1093/jncimonographs/djg014.

16. Fromme EK, Eilers KM, Mori $\mathrm{M}$, et al. How accurate is clinician reporting of chemotherapy adverse effects? A comparison with patient-reported symptoms from the Quality-of-Life Questionnaire C30. J Clin Oncol. 2004;22:3485-3490. https://doi.org/10.1093/jncimonographs/djg014.

17. Bäckström M, Mjörndal T, Dahlqvist R, et al. Spontaneous reporting of adverse drug reactions by nurses. Pharmacoepidemiology and Drug Safety.2003;11(8):647-650. https://doi.org/10.1002/pds.827.

18. Morrison-Griffiths S, Walley TJ, Park BK, et al. Reports of adverse drug reactions by nurses. Lancet. 2003;361(9366):1347-1348. https://doi.org/10.1016/S0140-6736(03)13043-7.

19. World Medical Association. (n.d.).WMA Declaration of Helsinki: Ethical principles for medical Research involving human subjects. Retrieved from http://www/wma.net/en/30publication/10polices/b3/.

20. Osoba D. Translating the science of patient-reported outcomes assessment into clinical practice. J Natl Cancer Inst Monogr. 2007;(37):5-11. https://doi.org/10.1093/jncimonographs/lgm002.

21. Cleeland CS, Mendoza TR, Wang XS, et al. Assessing symptom distress in cancer patients: the M.D. Anderson Symptom Inventory. Cancer. 2015;89(7):1634-1646. https://doi.org/10.1002/10970142(20001001)89:7<1634::AID-CNCR29>3.0.C0;2-V.

22. Cleeland CC.Symptom burden: multiple symptoms and their impact as patient reported outcomes. $J$ Natl Cancer Inst Monogr. 2007;(37): 16-21.20. https://doi.org/10.1093/jncimonographs/lgm005.

23. Lipscomb J, Reeve BB, Clauser SB et al. (2007). Patient-reported outcomes assessment in cancer trials: taking stock, moving forward. J Clin Oncol. 2007;(25):5133-5140. https://ascopubs.org/doi/10.1200/JC0.2007.12.4644.

24. Miyaji T, lioka Y, Kuroda Y, et al. Japanese translation and linguistic validation of the US National Cancer Institute's Patient-Reported Outcomes version of the Common Terminology Criteria for Adverse Events (PRO-CTCAE). Journal of Patient-Reported Outcomes. 2017;1(1):8-18. https://doi.org/10.1186/s41687-017-0012-7.

25. Hastings CE, Fisher CA, Mccabe MA. Clinical research nursing: a critical resource in the national research enterprise.Nursing Outlook. 2012;60(3):149-156. https://doi.org/10.1016/j.outlook.2011.10.003. 
26. Landis JR, Koch GG. (1977). The measurement of observer agreement for categorical data. Biometrics, . 1977;33(1):189-196. https://doi.org/10.2307/2529310.

\section{Figures}

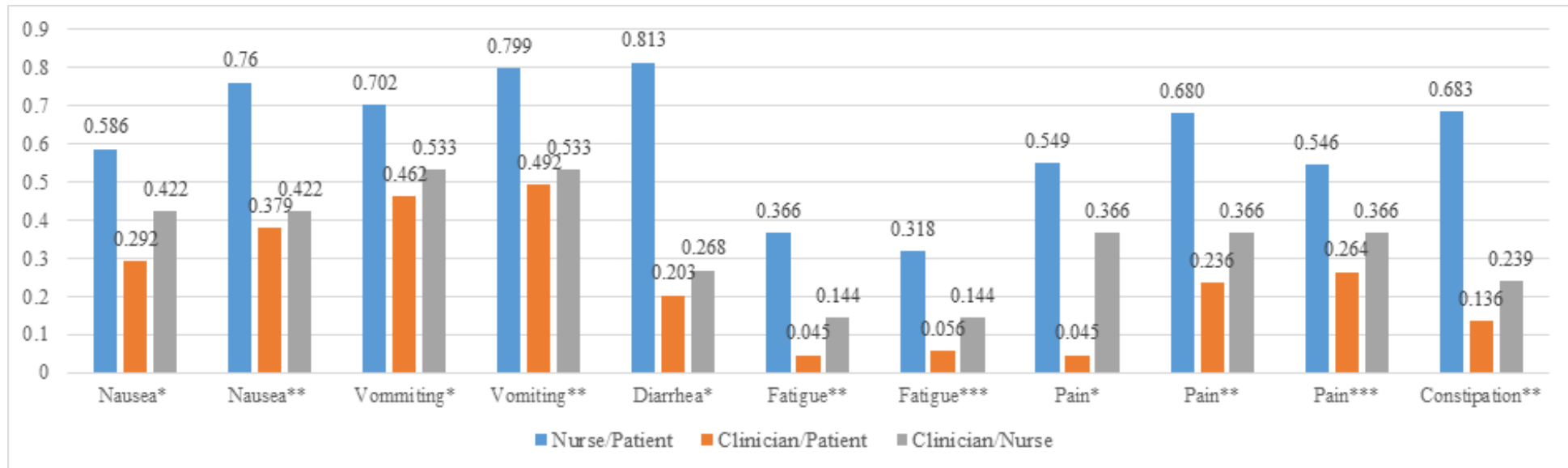

Figure 1

Consistency analysis of subjective AEs reporting rate between different pairs $k<0.000$ Poor agreement; $0.000 \leq k \leq 0.200$ Slight agreement; $0.210 \leq k \leq 0.400$ Fair agreement; $0.410 \leq k \leq 0.600$ Moderate agreement; $0.610 \leq k \leq 0.800$ Substantial agreement; $0.810 \leq k \leq 1.000$ Perfect agreement [26]

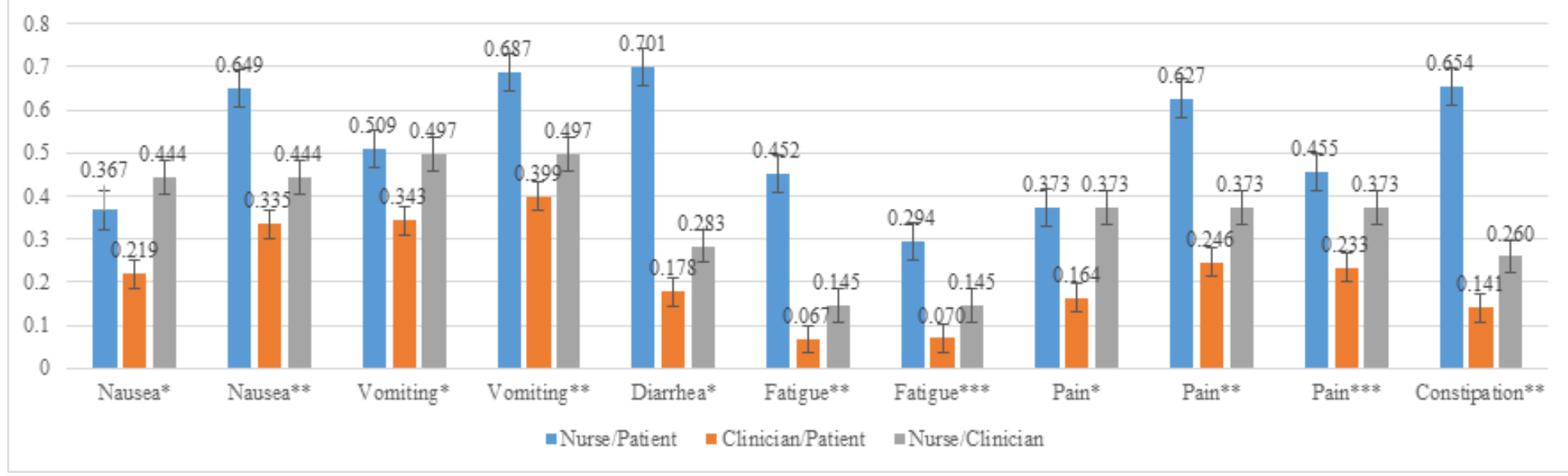

\section{Figure 2}

Consistency analysis between different pairs according to toxicity grade $(95 \% \mathrm{Cl})$ *indicating the Frequency of observed AEs; **indicating the Severity of observed AEs; $* * *$ indicating the interference of life of observed AEs 